December 1997

\title{
DECENTRALIZING EDGEWORTH EQUILIBRIA IN ECONOMIES WITH MANY COMMODITIES
}

\author{
Messaoud DEGHDAK * \\ Monique FLORENZANO **
}

$\mathrm{N}^{0} 9721$

\footnotetext{
* Département de Mathématiques, Université de Constantine, Algérie.

** CNRS-CEPREMAP, 140 rue du Chevaleret, 75013 Paris, France.
}

The main results of this paper were obtained during a visit of M. Deghdak to CEPREMAP. The hospitality of CEPREMAP is gratefully acknowledged. We thank C. Le Van for valuable discussions. 


\section{DECENTRALISATION DES EQUILIBRES D'EDGEWORTH POUR DES ECONOMIES AVEC UNE INFINITE DE BIENS}

\section{Résumé}

On appelle équilibre d'Edgeworth une allocation réalisable dont la répétition appartient au coeur de toutes les réplications de l'économie initiale. L'article établit, sous diverses hypothèses de propreté des préférences des consommateurs, des théorèmes d'équivalence entre équilibres d'Edgeworth et allocations d'équilibre compétitif pour une économie d'échange définie sur un espace de biens de dimension infinie. Les hypothèses sur la dualité biens - prix sont celles introduites par Mas-Colell et Richard, dans un papier publié en 1991 dans Journal of Economic Theory, pour rendre compte des propriétés minimales communes aux espaces spéciaux utilisés en Finance ou pour modéliser l'allocation intertemporelle des ressources ou la différenciation des produits. Il n'est fait par ailleurs aucune hypothèse de disposition sans coût des excédents et les préférences des consommateurs ne sont supposées ni transitives ni totales. Les hypothèses dites de propreté sur les préférences sont empruntées à des résultats récents d'existence de l'équilibre.

Mots clés : équilibre Walrasien, équilibre d'Edgeworth, équivalence coeur-équilibre, $F$-propreté, $E$-propreté, $M$-propreté.

\section{DECENTRALIZING EDGEWORTH EQUILIBRIA IN ECONOMIES WITH MANY COMMODITIES}

\footnotetext{
Abstract

This paper proves core-equivalence theorems for exchange economies without ordered preferences, defined on locally convex Riesz commodity spaces such that the price space is a lattice. Properness assumptions are borrowed from some recent equilibrium existence results.

Keywords : Walrasian equilibrium, Edgeworth equilibrium, core-equivalence theorem, $F$-properness, $E$-properness, $M$-properness.

JEL : C62, D51.
} 


\title{
Decentralizing Edgeworth equilibria in economies with many commodities
}

\author{
Messaoud Deghdak ${ }^{1}$ and Monique Florenzano ${ }^{2}$ \\ ${ }^{1}$ Département de Mathématiques, Université de Constantine, Algérie \\ 2 CNRS-CEPREMAP, 140 rue du Chevaleret, 75013 Paris, Franc
}

December 1997

\begin{abstract}
This paper proves core-equivalence theorems for exchange economies without ordered preferences, defined on locally convex Riesz commodity spaces such that the price space is a lattice. Properness assumptions are borrowed from some recent equilibrium existence results.
\end{abstract}

Keywords. Walrasian equilibrium, Edgeworth equilibrium, core-equivalence theorem, $F$-properness, $E$-properness, $M$-properness.

\section{Introduction}

This paper deals with Edgeworth's conjecture for economies with an infinite dimensional commodity space and without ordered preferences.

More precisely, let us follow Aliprantis, Brown and Burkinshaw [1990] and define Edgeworth equilibrium as any feasible (attainable) allocation which belongs to the core of every $r$-fold replica of the economy ( $r$ integer), when it is identified with an equal treatment allocation in the replica economy. The coincidence under suitable conditions between the set of Walrasian allocations and the set of Edgeworth equilibria for an economy with ordered preferences, defined in a finite dimensional commodity space, is a celebrated result of Debreu-Scarf [1963].

That Edgeworth equilibria exist under very mild conditions in an infinite dimensional framework was proved by Aliprantis, Brown and Burkinshaw [1987] for the ordered case, by Florenzano [1990] for the nonordered case. When the infinite dimensional commodity space is not of the $L_{\infty}$ type, decentralizing Edgeworth allocations by equilibrium prices so as to get Walrasian equilibria requires structural restrictions on the commodity space and properness assumptions on the preferences of the agents. Specifically, as well Aliprantis, Brown and Burkinshaw [1987] for the ordered case as Florenzano [1990] for the nonordered case

\footnotetext{
${ }^{1}$ The main results of this paper were obtained during a visit of M. Deghdak to CEPREMAP. The hospitality of CEPREMAP is gratefully acknowledged. We thank C. Le Van for valuable discussions.
} 
assume a topological vector lattice structure of the commodity space and use uniform properness conditions, first introduced by Mas-Colell [1986] and adapted to the nonordered case by Yannelis and Zame [1986]. It should not be surprising to meet here some by now classical conditions sufficient for the existence of Walrasian equilibria in an infinite dimensional framework, since adding a core equivalence theorem to the existence of Edgeworth equilibria is another way for getting an equilibrium existence theorem.

Since the seminal paper of Mas-Colell [1986], first circulated in 1983, the classical conditions for the existence of Walrasian equilibrium have been weakened in two directions.

The first improvement concerns the topological vector lattice stucture of the commodity space. After Bewley [1972], Banach lattices could seem natural commodity spaces for modelling several problems of intertemporal allocation of resources in Economics or in Finance. However, the topology considered on the commodity space determines the price space, as far as one looks for continuous equilibrium prices. To require the continuity of the lattice operations may rule out some economically meaningful commodity-price duality. Specifically, it is in order to treat the equilibrium existence problem in some models of differentiation of commodities or of intertemporal consumption that Mas-Colell and Richard [1991] have replaced this requirement by the weaker one of a lattice structure on the price space. Even if this framework leaves out of its scope some commodity-price dualities of economic interest (a detailed discussion on relevant commodity-price dualities can be found in Mas-Colell and Zame [1991]), it has been used after Mas-Colell and Richard in several equilibium existence proofs : Richard [1989], Podczeck [1996], Marakulin [1995].

On the other hand, whatever be the topology considered on the commodity space, properness assumptions on the preferences of the consumers are strong assumptions (and weaker is the topology, stronger are these assumptions). As known since Mas-Colell [1986], some pointwise properness cannot be dispensed with. However, the uniformity of properness is too strong and has been found incompatible with some assumptions on utility functions, current in Finance. The uniformity has been seriously weakened by Zame [1987] and completely replaced by an assumption of pointwise properness at some particular allocations by Araujo and Monteiro [1989], Duffie and Zame [1989], in the particular case where the total endowment of the economy is a strictly positive element of the commodity space. This result is extended by Podczeck to the nonordered case and proved without any monotonicity assumption. For the general case where the total endowment is not a quasi-interior element of the commodity space (specially, if this one has no quasi-interior element), Podczeck introduces a properness concept, called $E$-properness, stronger than pointwise properness but weaker than uniform properness. In the same general case, Marakulin keeps the uniformity but defines a properness, called here $M$-properness, weaker than the original one. 
The purpose of this paper is to reconsider Florenzano [1990] at the light of the previously quoted equilibrium existence results. As Podczeck and Marakulin, we make on the commodity space the assumptions of Mas-Colell and Richard [1991], Richard [1989]. In this framework, using different properness assumptions, we look for prices which decentralize Edgeworth equilibria. The coincidence is complete between the set of Edgeworth equilibria and the set of Walrasian allocations, when the properness assumptions are the one of Podczeck. Under assumptions of uniform $M$-properness, we get a result similar to the one of Florenzano [1990]. We define an hypothetical economy which has Edgeworth equilibria and we prove the equivalence between Edgeworth equilibria of this economy and Walrasian allocations of the original one. All results are proved for nonordered preferences. When preferences are ordered, all the Edgeworth equilibria of the original economy are decentralized.

These results have their own interest. It is worth noticing that a by-product of these core equivalence theorems is another proof of the Podczeck equilibrium existence results and an extension of the result of Marakulin.

The paper is organized as follows. Section 2 describes the model and posits the main assumptions. Section 3 comments on different properness concepts and their reciprocal relations. Introducing properness assumptions, Section 4 presents and proves core equivalence theorems. Section 5 is devoted to a comparison between the results proved in the paper and the corresponding results already proved or which could be proved under the stronger assumption that the commodity space is a topological vector lattice. An appendix gives a sketch of a proof ommitted in the text.

\section{The model}

Let us consider an exchange economy, $\mathcal{E}=\left(\left\langle L, L^{\prime}\right\rangle,\left(X_{i}, P_{i}, \omega_{i}\right)_{i \in I}\right)$, where

- $\left\langle L, L^{\prime}\right\rangle$, interpreted as the commodity-price duality, is a pair of vector spaces and an associated bilinear functional $\langle\cdot, \cdot\rangle: L \times L^{\prime} \rightarrow R$ that separates points

- $I=\{1, \ldots, m\}$ is a finite set of consumers

- Each consumer $i \in I$ has a consumption set $X_{i} \subset L$, an initial endowment $\omega_{i} \in X_{i}$ and a (strict) preference relation $P_{i}: X_{i} \rightarrow X_{i}$.

The following classical definitions adapt to the nontransitive context usual ones given when each $P_{i}$ is the asymetric part of a reflexive, transitive and complete relation $R_{i}$ on $X_{i}$.

Let $\omega=\sum_{i \in I} \omega_{i}$. An allocation $x \in \prod_{i \in I} X_{i}$ is feasible if $\sum_{i \in I} x_{i}=\omega$. A quasiequilibrium is a feasible allocation and a price $p \in L^{\prime}, p \neq 0$, such that for every $i \in I$ : 
$-p \cdot x_{i} \leq p \cdot \omega_{i}$

- if $y \in P_{i}\left(x_{i}\right)$, then $p \cdot y \geq p \cdot \omega_{i}$.

A quasi-equilibrium such that for every $i, y \in P_{i}\left(x_{i}\right)$ actually implies $p \cdot y>p \cdot \omega_{i}$ is a Walrasian equilibrium. We will denote by $\mathcal{W}(\mathcal{E})$ the set of feasible allocations that are equilibrium allocations for some $p \in L^{\prime}$.

Now, let us call coalition any nonempty subset of $I$. A coalition $B$ blocks a feasible allocation $\bar{x}$ if there exists $\left(x_{i}\right)_{i \in B} \in \prod_{i \in B} X_{i}$ such that $\sum_{i \in B} x_{i}=\sum_{i \in B} \omega_{i}$ and $x_{i} \in P_{i}\left(\bar{x}_{i}\right) \forall i \in B$. The core of $\mathcal{E}, \mathcal{C}(\mathcal{E})$, is the set of all feasible allocations which are blocked by no coalition.

Then let $r$ be any positive integer. The $r$-fold replica of $\mathcal{E}$ is a new exchange economy, $\mathcal{E}^{r}=\left(\left\langle L, L^{\prime}\right\rangle,\left(X_{i q}, P_{i q}, \omega_{i q}\right)_{\substack{i=1, \ldots, m \\ q=1, \ldots, r}}\right)$, with the same commodity-price duality and $m r$ consumers, indexed by $i q$, such that for every $i, X_{i q}=X_{i}$, $P_{i q}=P_{i}$ and $\omega_{i q}=\omega_{i}$. An allocation in $\mathcal{E}^{r}$ has the equal treatment property if $x_{i s}=x_{i t}$ for all $i=1, \cdots, m, s$ and $t \in\{1, \cdots, r\}$. Noticing that the total endowment in $\mathcal{E}^{r}$ is precisely $r \omega$, feasible equal treatment allocations of $\mathcal{E}^{r}$ can be identified with feasible allocations of the original economy. Following Aliprantis et al. (1987), we say that a feasible allocation $\bar{x}$ of $\mathcal{E}$ is an Edgeworth equilibrium, and we write $\bar{x} \in \mathcal{C}^{e}(\mathcal{E})$, if $\bar{x}$ belongs to the core of every replication of $\mathcal{E}$.

Finally, let $T=[0,1]^{I} \backslash\{0\}$ and let $T_{Q}$ be the set of all $t=\left(t_{i}\right)_{i \in I} \in T$ such that each $t_{i}$ is a rational number. If we assume that each $X_{i}$ is convex and that for each $i$, for every $x_{i} \in X_{i}, P_{i}\left(x_{i}\right)$ is convex (both assumptions will be made later), it is easily seen (for details, see Florenzano [1990]) that $\bar{x} \in \mathcal{C}^{e}(\mathcal{E})$ if and only if there exists no $t=\left(t_{i}\right)_{i \in I} \in T_{Q}$ and no $x_{t} \in \prod_{t_{i}>0} X_{i}$ with $\sum_{t_{i}>0} t_{i} x_{i t}=\sum_{i \in I} t_{i} \omega_{i}$ and $x_{i t} \in P_{i}\left(\bar{x}_{i}\right) \forall i: t_{i}>0$. Here $t_{i}$ can be understood as the rate of participation of consumers of type $i$ in a coalition of some r-fold replica of $\mathcal{E}$. Allowing, as Aubin [1979], the rates of participation of consumers of type $i$ to take all values in the real interval $[0,1]$, we say that a feasible allocation $\bar{x}$ belongs to the fuzzy core of $\mathcal{E}$, and we write $\bar{x} \in \mathcal{C}^{f}(\mathcal{E})$, if there exist no $t=\left(t_{i}\right)_{i \in I} \in T$ and no $x_{t} \in \prod_{t_{i}>0} X_{i}$ with $\sum_{t_{i}>0} t_{i} x_{i t}=\sum_{i \in I} t_{i} \omega_{i}$ and $x_{i t} \in P_{i}\left(\bar{x}_{i}\right) \forall i: t_{i}>0$.

As a straightforward consequence of the previous definitions, $\mathcal{W}(\mathcal{E}) \subset \mathcal{C}^{f}(\mathcal{E}) \subset$ $\mathcal{C}^{e}(\mathcal{E}) \subset \mathcal{C}(E)$. $\mathcal{E}$ :

We will maintain in the whole paper the following assumptions on the economy

A.1 $L$ is a vector lattice (Riesz space) endowed with a Hausdorff locally convex topology $\tau$ such that $L_{+}$is $\tau$-closed

A.2 $L^{\prime}=(L, \tau)^{\prime}$, the topological dual of $L$, and $L^{\prime}$ is a vector sublattice of $L^{\sim}$, the order-dual of $L$

A. $3 \forall i \in I, X_{i}=L_{+}$and $\omega_{i} \in X_{i}$ 
A.4 $\forall i \in I, \forall x_{i} \in L_{+}, P_{i}\left(x_{i}\right)$ is convex and $x_{i} \notin P_{i}\left(x_{i}\right)$

A. $5 \forall i \in I, \forall x_{i} \in L_{+} P_{i}\left(x_{i}\right)$ is a relatively $\tau$-open subset of $L_{+}$

A.6 There exists a Hausdorff vector space topology $\sigma$ on $L$ such that the order interval $[0, \omega]$ is $\sigma$-compact and such that, for every $i \in I$ and for every $x_{i} \in L_{+}$, $P_{i}^{-1}\left(x_{i}\right) \stackrel{\text { def }}{=}\left\{y_{i} \in L_{+} \mid x_{i} \in P_{i}\left(y_{i}\right)\right\}$ is $\sigma$-open in $L_{+}$.

The assumptions A.1 and A.2 on the commodity-price duality are exactly those done in Mas-Colell and Richard [1991], Marakulin [1995], Richard [1989], Podczeck [1996]. Recall that they weaken the standard assumption that the topology $\tau$ is locally solid by assuming a lattice structure on the price space which obtains automatically if the commodity space is a topological vector lattice.

Assumptions A.3 - A.6 are classical for economies with an infinite dimensional commodity-space. It follows from Propositions 3 and 4 in Florenzano [1990] that, under A.1 and A.3 - A.6, the set of Edgeworth equilibria, $\mathcal{C}^{e}(\mathcal{E})$, is nonempty and coincides with the fuzzy core, $\mathcal{C}^{f}(\mathcal{E})$.

\section{Properness}

For decentralizing Edgeworth equilibria when the commodity space $L$ is infinite dimensional, properness assumptions compensate the fact that the consumption sets may have empty interior. Since Mas-Colell [1986], several such assumptions have been used in the literature in order to obtain an equilibrium existence theorem. For a preference relation $P$ on $L_{+}$, we will consider in this paper three conditions, hereafter defined, which all explicitely rely on the topology $\tau$ considered on $L$. In view of Assumption A.1 on $L$, properness 0-neighborhoods used in these definitions will always be assumed to be convex and circled.

Definition $3.1 v \in L$ is extremely desirable for $P$ on $W \subset L_{+}$if there exist an open 0-neighborhood $V$ and, for each $u \in V$, a real number $\bar{\lambda}_{u}>0$ such that if $x \in W$, if $u \in V$ and if $0<\lambda<\bar{\lambda}_{u}$, then $x+\lambda(v-u) \in P(x)$, provided $x+\lambda(v-u) \in L_{+}$.

$P$ is $F$-proper on $W$ if there exists some $v \in L$, extremely desirable for $P$ on $W$. $P, F$-proper on $\{x\}$, is said to be F-proper at $x$.

Definition 3.1 goes to Yannelis and Zame [1986]. Let $C$ be the open set, $C=$ $\left\{\lambda(v-u) \mid u \in V, 0<\lambda<\bar{\lambda}_{u}\right\}$. As well known, extreme desirability of $v$ means that at every $x \in W$ the portion of the forward set $\{x\}+C$ which belongs to $L_{+}$ is contained in the set of the points $P$-preferred to $x$, so that in $F$-properness, $F$ stands for "forward".

It is worth noticing that if $P$ is the asymmetric part of a reflexive, transitive and complete relation on $L_{+}$(for short, in a transitive or ordered context), then 
the property defining extreme desirability of $v$ on $L_{+}$is satisfied for every $\lambda>$ 0 . In view of this remark, it is easily verified that $F$-properness on $L_{+}$is the nontransitive version of uniform properness on $L_{+}$as defined by Mas-Colell [1986] in the transitive context. However, if $W \neq L_{+}, F$-properness on $W$ and MasColell (uniform) properness on $W$ are incomparable conditions. It should also be emphasized that $P$ may be $F$-proper at every $x$ of some subset $W$ of $L_{+}$and not $F$-proper on $W$. In other words, $F$-properness at every point of some subset of $L_{+}$does not entail any uniformity of the properness constants.

In a transitive context, $F$-properness of preferences of consumers at every component of an individually rational and Pareto optimal allocation has been used by Araujo and Monteiro [1989] to prove the existence of a (free-disposal) quasi-equilibrium in an exchange economy satisfying Assumptions A.3 - A.6, defined on a locally convex-solid vector lattice containing as a dense subspace the order ideal, $L(\omega)$, generated in $L$ by the total endowment $\omega$. Under Assumptions A. 1 and A.2 on the commodity-price duality and with the same density condition of $L(\omega)$ in $L$, the result is got by Podczeck [1996], without disposal, and extended to a nontransitive context.

Definition 3.2 Let $x \in L_{+}$and let $K$ be a linear subspace of $L$ with $x \in K . P$ is $E$-proper at $x$ relative to $K$ if there is some $v_{x} \in L$, some 0-neighborhood $V_{x}$ and some subset $A_{x}$ of $K$ which is radial ${ }^{2}$ at $x$ (in $K$ ) such that

(a) $x+\alpha v_{x} \in P(x)$ for every sufficiently small real number $\alpha>0$

(b) $\left(P(x)+\Gamma_{x}\right) \cap L_{+} \cap A \subset P(x)$, where $\Gamma_{x}$ is the open cone $\Gamma_{x}=\left\{\lambda\left(v_{x}-u\right) \mid\right.$ $\left.0<\lambda, u \in V_{x}\right\}$.

Definition 3.2 is set and discussed in Podczeck [1996]. Obviously, in a transitive context, if $P$ is $F$-proper on $L_{+}$, then $P$ is $E$-proper relative to $L$ at every $x \in L_{+}$ with the same (uniform) properness constants. An example given by Podczeck shows that the reverse implication does not hold.

For any subset $B$ of $L$, let $\bar{B}^{\tau}$ denote the $\tau$-closure of $B$. Actually, the condition (b) in Definition 3.2 implies that $\left(\overline{P(x)}^{\top}+\Gamma\right) \cap L_{+} \cap A \subset P(x)$. As the condition (a) implies that $x \in \overline{P(x)}^{\tau}$, it follows that for a preference relation $P$, $E$-properness at $x$ relative to $L$ strengthens $F$-properness at $x$.

As proved in Podczeck, if $L$ (satisfying A.1) has a positive cone with a nonempty $\tau$-interior, then every open and convex valued preference relation is as well $F$-proper as $E$-proper relative to $L$ at every point $x$ of $L_{+}$which is a point of local nonsatiation. More generally, under Assumption A.1 on $L, P$ is as well $F$-proper as $E$-proper relative to $L$ at $x \in L_{+}$, provided $x$ is a point of local nonsatiation and $P(x)$ can be convex open extended (i.e. $P(x)=\widetilde{P}(x) \cap L_{+}$for some convex and open subset $\widetilde{P}(x)$ of $L)$.

\footnotetext{
${ }^{2}$ If $A$ is a subset of a vector space $K$, then $A$ is called radial (absorbing) at a point $x \in A$ if for each $y \in K$, there exists a real number $\bar{\lambda}, 0<\bar{\lambda} \leq 1$, such that $(1-\lambda) x+\lambda y \in A$ for every $\lambda$ with $0 \leq \lambda \leq \bar{\lambda}$.
} 
The $E$-properness relative to $L(\omega)$ of preferences of consumers at every component $x$ of an individually and Pareto optimal allocation, with properness vectors $v_{x_{i}}$ satisfying $x_{i}+v_{x_{i}} \in L(\omega)_{+}$, was used by Podczeck [1996] to get the existence of a quasiequilibrium for an exchange economy satisfying A.1-A.6. This result extends to nontransitive (and non-monotone) preferences the Mas-Colell-Richard [1991] equilibrium existence theorem.

Definition $3.3 P$ is $M$-proper on $W \subset L_{+}$if there exist $v \in L_{+}$and an open 0 -neigbborhood $V$ such that if $x \in W$, then $x \notin P(x)+\Gamma$, where $\Gamma$ is the open cone $\Gamma=\{\lambda(v-u) \mid 0<\lambda, u \in V\}$.

$P$ is uniformly $M$-proper if $W=L_{+}$in the previous definition.

Definition 3.3 was set by Marakulin [1995]. For this reason, $M$ stands for Marakulin in the previous definition. In a transitive context, uniform $M$-proper ness slightly weakens uniform properness as defined by Mas-Colell [1986] and used in Mas-Colell-Richard [1991]. In a transitive context as in a nontransitive context, the relations we establish between uniform $M$-properness and the previous properness concepts heavily rely on the uniformity of $M$-properness.

Proposition 3.1 Assume that $P$, uniformly $M$-proper, is the asymmetric part of a complete preorder on $L_{+}$. Then $P$ is $F$-proper and $E$-proper relative to $L$ at every point $x$ which is a point of local nonsatiation for $P$, with the same (uniform) properness vector.

Proof. From transitivity and completeness of the preorder, one easily deduces that for every $x \in L_{+},(P(x)+\Gamma) \cap L_{+} \subset P(x)$, which is Condition (b) in Definition 3.2. Then if $x$ is a point of local nonsatiation of $P$, from $\left(\overline{P(x)}^{\tau}+\Gamma\right) \cap L_{+} \subset$ $P(x)$, one deduces $(\{x\}: \Gamma) \cap L_{+} \subset P(x)$, which proves as well Condition (a) of $E$-properness as $F$-properness of $P$ at $x$.

Proposition 3.2 Assume that $P$ is uniformly $M$-proper and let $Q$ be the preference relation defined on $L_{+}$by $Q(x)=(P(x)+\Gamma) \cap L_{+}$. Then, $Q$ is F-proper and $E$-proper relative to $L$ at every point $x$ which is a point of local nonsatiation for $P$, with the same (uniform) properness vector.

Proof. Indeed, for every $x \in L_{+},(Q(x)+\Gamma) \cap L_{+}=\left(\left((P(x)+\Gamma) \cap L_{+}\right)+\right.$ $\Gamma) \cap L_{+} \subset(P(x)+\Gamma+\Gamma) \cap L_{+} \subset(P(x)+\Gamma) \cap L_{+}$, which proves condition (b) of Definition 3.2. From $(Q(x)+\Gamma) \cap L_{+} \subset Q(x)$, one deduces $\left(\overline{Q(x)}^{\tau}+\Gamma\right) \cap L_{+} \subset$ $Q(x)$. Then, if $x \in \overline{P(x)}^{\top}, x \in \overline{Q(x)}^{T}$ and $(x+\Gamma) \cap L_{+} \subset Q(x)$, which proves condition (a) of Definition 3.2 and $F$-properness of $Q$ at $x$. 
The uniform $M$-properness with $\omega$ as properness vector of preferences of consumers, together with their monotonicity $\left(P_{i}(x)+L_{+} \subset P_{i}(x)\right)$ and local nonsatiation at every point of $L_{+}$was used by Marakulin [1995] to prove the existence of a quasi-equilibrium. At the end of Section 4, we will get an analogous result without monotonicity.

\section{From Edgeworth equilibria to quasi-equilibria}

In order to get what is generally called an equivalence theorem, we now start with an Edgeworth equilibrium $\bar{x}=\left(\bar{x}_{i}\right)_{i \in I}$ of $\mathcal{E}$. As already noticed, under Assumptions A.1 and A.3 - A.6 on $\mathcal{E}$, such elements exist and belong to the fuzzy core $\mathcal{C}^{f}(\mathcal{E})$.

Let us call $\mathcal{E}_{\mid L(\omega)}$ the economy, deduced from $\mathcal{E}$, where consumers are restricted to the order-ideal $L(\omega)$ generated by the total endowment $\omega$ as commodity space. As well known, $L(\omega)$ can be endowed with the Riesz norm $\|x\|_{\omega}=\inf \{\lambda>0 \mid$ $|x| \leq \lambda \omega\}$, so that the unit-ball is the order interval $[-\omega,+\omega]$. Moreover, as noticed by Podczeck, the fact that on $L(\omega)$, the norm topology is finer than the topology induced by $\tau$ still holds true when Assumptions A.1 and A.2 replace the classical assumption that $L$ is a locally convex-solid Riesz space. We consider for $\mathcal{E}_{\mid L(\omega)}$ the commodity-price duality $\left\langle\left(L(\omega),\|\cdot\|_{\omega}\right),\left(L(\omega),\|\cdot\|_{\omega}\right)^{\prime}\right\rangle$. In what follows, we will write $L(\omega)^{\prime}$ for $\left(L(\omega),\|\cdot\|_{\omega}\right)^{\prime}$.

The next proposition generalizes at several instances, and in particular to a nontransitive context, Theorem 3.4.18 of Aliprantis, Brown and Burkinshaw [1990]. It proves the existence of $\bar{p} \in L(\omega)^{\prime}$ such that $(\bar{x}, \bar{p})$ is a quasi-equilibrium of $\mathcal{E}_{\mid L(\omega)}$, provided that each component $\bar{x}_{i}$ of $\bar{x}$ satisfies in $\mathcal{E}_{\mid L(\omega)}$ local nonsatiation of preference of consumer $i$.

Proposition 4.1 Under Assumptions A.1, A.3-A.5 on $\mathcal{E}$, if $\bar{x} \in \mathcal{C}^{f}(\mathcal{E})$ and if, for each $i \in I, \bar{x}_{i} \in \bar{P}_{i}\left(\bar{x}_{i}\right) \cap L(\omega)\|\cdot\|_{\omega}$, the $\|\cdot\|_{\omega}$-closure of $P_{i}\left(\bar{x}_{i}\right) \cap L(\omega)$, then there exists $\bar{p} \in L(\omega)^{\prime}, \bar{p} \neq 0$, such that $(\bar{x}, \bar{p})$ is a quasi-equilibrium of $\mathcal{E}_{\mid L(\omega)}$.

Moreover, $\inf \left\{\bar{p} \cdot z \mid z \in L(\omega)_{+}\right\}<\bar{p} \cdot \omega_{i}$ for some $i \in I$.

Proof. Let $G \stackrel{\text { def }}{=} \operatorname{co}\left(\bigcup_{i \in I}\left(\left(P_{i}\left(\bar{x}_{i}\right) \bigcap L(\omega)\right)-\omega_{i}\right)\right)$. In view of Assumptions A.4, A.5 and the previous remark on the topology of the Riesz norm on $L(\omega)$, each $P_{i}\left(\bar{x}_{i}\right)$ is $\|\cdot\|_{\omega}$-open in $L(\omega)_{+}$and $G$ is a convex subset of $L(\omega)$ with a nonempty $\|\cdot\|_{\omega}$-interior. Moreover, $\bar{x} \in \mathcal{C}^{f}(\mathcal{E})$ implies $0 \notin G$. It then follows from the classical separation theorem that there exists $\bar{p} \in L(\omega)^{\prime}, \bar{p} \neq 0$, such that $x_{i} \in$ $P_{i}\left(\bar{x}_{i}\right) \cap L(\omega) \Rightarrow \bar{p} \cdot x_{i} \geq \bar{p} \cdot \omega_{i}$. It follows from the local nonsatiation (in $L(\omega)_{+}$) of each $P_{i}$ at $\bar{x}_{i}$ that $\bar{p} \cdot \bar{x}_{i}=\bar{p} \cdot \omega_{i}$, for every $i$.

Finally, if for every $i \in I, \inf \left\{\bar{p} \cdot z \mid z \in L(\omega)_{+}\right\} \geq \bar{p} \cdot \omega_{i}$, then from $\frac{\omega}{m} \in \operatorname{int}_{\|\cdot\|_{\omega}}\left(L(\omega)_{+}\right)$, one deduces that for some $\varepsilon>0, \bar{p} \cdot\left(\frac{\omega}{m}+\varepsilon[-\omega,+\omega]\right) \geq \bar{p} \cdot \omega_{i}$ 
holds for every $i \in I$. By summing on $i$, one gets $m \varepsilon \bar{p} \cdot[-\omega,+\omega] \geq 0$, which contradicts $\bar{p} \neq 0$.

The last statement of Proposition 4.1 means that $(\bar{x}, \bar{p})$ is what is called by Podczeck [1996] a non-trivial quasi-equilibrium of $\mathcal{E}_{\mid L(\omega)}$. Under some additional irreducibility assumption on $\mathcal{E}_{\mid L(\omega)}$ (see Florenzano [1982] for a discussion of some irreducibility concepts), $\inf \left\{\bar{p} \cdot z \mid z \in L(\omega)_{+}\right\}<\bar{p} \cdot \omega_{i}$ holds for every $i$ and, in view of $\mathrm{A} .5,(\bar{x}, \bar{p})$ is a Walrasian equilibrium of $\mathcal{E}_{\mid L(\omega)}$.

Our next step is to introduce properness assumptions for each $P_{i}$ at the component $\bar{x}_{i}$ of $\bar{x}$ in order to guarantee the local nonsatiation of $P_{i}$ at $\bar{x}_{i}$ assumed in Proposition 4.1 and to extend the price $\bar{p}$ got in this proposition to a $\tau$-continuous linear functional $\bar{\pi}$, defined on the whole commodity space $L$, such that $(\bar{x}, \bar{\pi})$ is a quasi-equilibrium of $\mathcal{E}$. The way of doing this extension, as far we deal with $F$ or $E$-properness assumptions, is essentially borrowed from Podczeck [1996].

Proposition 4.2 Under Assumptions $A .1-A .5$ on $\mathcal{E}$, if $\bar{x} \in \mathcal{C}^{f}(\mathcal{E})$, suppose that

- either $L(\omega)$ is $\tau$-dense in $L$ and each $P_{i}$ is $F$-proper at $\bar{x}_{i}$, with a properness vector $v_{i}$ satisfying $\bar{x}_{i}+v_{i} \in L_{+}$

- or each $P_{i}$ is $E$-proper at $\bar{x}_{i}$ relative to $L(\omega)$, with a properness vector satisfying $\bar{x}_{i}+v_{i} \in L(\omega)_{+}$.

Then there exists $\bar{\pi} \in L^{\prime}, \dot{\bar{\pi}} \neq 0$, such that $(\bar{x}, \bar{\pi})$ is a non-trivial quasi-equilibrium of $\mathcal{E}$.

Proof. We first verify the local nonsatiation of each $P_{i}$ at $\bar{x}_{i}$.

Suppose $L(\omega)$ is $\tau$-dense in $L$ and $P_{i}$ is $F$-proper at $\bar{x}_{i}$ with a properness vector $v_{i}$ such that $\bar{x}_{i}+v_{i} \in L_{+}$. We first remark that, in view of A.2, $L(\omega)_{+}$is $\tau$-dense in $L_{+}$(Lemma 3 in Podczeck(1996)). Then let $v_{i}^{\prime}$ be such that $\bar{x}_{i}+v_{i}^{\prime} \in L(\omega)_{+}$ and $v_{i}^{\prime}-v_{i} \in V_{i}$, where $V_{i}$ is the properness 0-neighborhood. Recalling that $\bar{x}_{i} \in L(\omega)_{+}$, we have $\bar{x}_{i}+\lambda v_{i}^{\prime} \in \bar{x}_{i}+\lambda\left(v_{i}+V_{i}\right)$, so that, for every positive and small enough $\lambda, \bar{x}_{i}+\lambda v_{i}^{\prime} \in P_{i}\left(\bar{x}_{i}\right) \cap L(\omega)_{+}$. This proves that $\bar{x}_{i}$ belongs to the $\|\cdot\|_{\omega}$-closure of $P_{i}\left(\bar{x}_{i}\right) \cap L(\omega)_{+}$.

Suppose now that $P_{i}$ is $E$-proper at $\bar{x}_{i}$ relative to $L(\omega)$ with a properness vector $v_{i}$ satisfying $\bar{x}_{i}+v_{i} \in L(\omega)_{+}$. From the condition (a) in Definition 3.2, it follows that for every positive and small enough $\lambda, \bar{x}_{i}+\lambda v_{i} \in P_{i}\left(\bar{x}_{i}\right) \cap L(\omega)_{+}$. Then, obviously, $\bar{x}_{i}$ belongs to the $\|\cdot\|_{\omega}$-closure of $P_{i}\left(\bar{x}_{i}\right) \cap L(\omega)_{+}$.

Applying now Lemma 4 in Podczeck $(1996)^{3}$, we get $\bar{\pi} \in L^{\prime}$, extending $\bar{p}$, such that $(\bar{x}, \bar{\pi})$ is a quasi-equilibrium of $\mathcal{E}$. Since $(\bar{x}, \bar{p})$ was non-trivial, $(\bar{x}, \bar{\pi})$ is also

\footnotetext{
${ }^{3}$ In order to keep this paper self-contained, we indicate in the appendix a sketch of the proof of this lemma.
} 
non-trivial.

Let us now consider on the economy $\mathcal{E}$ the following assumptions :

A.7 $L(\omega)$ is $\tau$-dense in $L$ and each $P_{i}$ is $F$-proper at every component $\bar{x}_{i}$ of an Edgeworth equilibrium $\bar{x}$, with a properness vector $v_{i}$ satisfying $\bar{x}_{i}+v_{i} \in L_{+}$

A.8 Each $P_{i}$ is $E$-proper relative to $L(\omega)$ at every component $\bar{x}_{i}$ of an Edgeworth equilibrium $\bar{x}$, with a properness vector satisfying $\bar{x}_{i}+v_{i} \in L(\omega)_{+}$.

It follows from Proposition 4.2 that under Assumptions A.1 - A.5 and some additional irreducibility assumption on $\mathcal{E}$ (a priori weaker than an irreducibility assumption on $\mathcal{E}_{\mid L(\omega)}$, each one of the assumptions A.7 and A.8 is sufficiently strong to guarantee a complete coincidence between the set of Walrasian equilibrium allocations, $\mathcal{W}(\mathcal{E})$, and the set of Edgeworth equilibria, $\mathcal{C}^{e}(\mathcal{E})$. Obviously, adding Assumption A.6 guarantees the nonemptiness of each one of these sets.

This is no longer true when we deal with uniform $M$-properness. In the next propositions, using uniform $M$-properness of preferences for each consumer and a classical local nonsatiation assumption of each $P_{i}$ in every component of an attainable allocation, we show that some allocations (proved to exist) in $\mathcal{C}^{e}(\mathcal{E})$ can be decentralized by a price system as quasi-equilibria of $\mathcal{E}$.

More precisely, let us consider on the economy $\mathcal{E}$ the following assumptions :

A.9 Each $P_{i}$ is uniformly $M$-proper with a (uniform) properness vector $v_{i} \in$ $L(\omega)_{+}$

A.10 For every attainable allocation $\bar{x}$ and for each $i \in I, \bar{x}_{i} \in \overline{P_{i}\left(\bar{x}_{i}\right)}$, the $\tau$-closure of $P_{i}\left(\bar{x}_{i}\right)$ in $L$.

For each $i$, let $\Gamma_{i}$ denote the (uniform) properness cone $\Gamma_{i}=\left\{\lambda\left(v_{i}-u\right)|\lambda\rangle\right.$ $\left.0, u \in V_{i}\right\}$ and let $Q_{i}$ be the relation on $L_{+}$defined by $Q_{i}\left(x_{i}\right)=\left(P_{i}\left(x_{i}\right)+\right.$ $\left.\Gamma_{i}\right) \cap L_{+}$. Assumption A.9 insures that each $Q_{i}$ satisfies irreflexivity, i.e. that each $Q_{i}$ is a (strict) preference on $L_{+}$. We now consider the economy $\mathcal{E}^{\Gamma}=$ $\left(\left\langle L, L^{\prime}\right\rangle,\left(X_{i}, Q_{i}, \omega_{i}\right)_{i \in I}\right)$ that we associate with the original economy $\mathcal{E}=\left(\left\langle L, L^{\prime}\right\rangle,\left(X_{i}, P_{i}, \omega_{i}\right)_{i \in I}\right)$.

Proposition 4.3 Under Assumptions A.1, A.3 - A.6 and A.9 on the economy $\mathcal{E}$, the fuzzy core of $\mathcal{E}^{\Gamma}, \mathcal{C}^{f}\left(\mathcal{E}^{\Gamma}\right)$ is nonempty and coincides with the set of Edgeworth equilibria of $\mathcal{E}^{\Gamma}, \mathcal{C}^{e}\left(\mathcal{E}^{\Gamma}\right)$.

Proof. We have only to verify the assumptions A.4 - A.6 on $\mathcal{E}^{\Gamma}$, using A.9 and the corresponding assumptions on $\mathcal{E}$.

For every $x_{i} \in L_{+}$, each $Q_{i}\left(x_{i}\right)$ is convex and $\tau$-open in $L_{+}$, by construction. $x_{i} \notin Q_{i}\left(x_{i}\right)$ follows from A.9. Suppose now that for some net $\left(z^{\alpha}\right) \subset L_{+}$, we have $z^{\alpha} \notin Q_{i}^{-1}\left(x_{i}\right), \forall \alpha$ and $z^{\alpha} \stackrel{\sigma}{\longrightarrow} z \in L_{+}$. If $z \in Q_{i}^{-1}\left(x_{i}\right)$, then $x_{i} \in Q_{i}(z)$, i.e. 
$x_{i}=t_{i}+\lambda\left(v_{i}-u\right)$ for some $t_{i} \in P_{i}(z), \lambda>0, u \in V_{i}$. Then, in view of A.6 on $\mathcal{E}$, for large enough $\alpha, t_{i} \in P_{i}\left(z^{\alpha}\right)$ and $x_{i} \in Q_{i}\left(z^{\alpha}\right)$, which contradicts $z^{\alpha} \notin Q_{i}^{-1}\left(x_{i}\right)$. From this, one deduces $z \notin Q_{i}^{-1}\left(x_{i}\right)$, which proves that $Q_{i}^{-1}\left(x_{i}\right)$ is $\sigma$-open in $L_{+}$.

Proposition 4.4 Under Assumptions A.1 - A.5, A.9 and A.10, let $\bar{x} \in \mathcal{C}^{f}\left(\mathcal{E}^{\Gamma}\right)$. Then there exists $\bar{\pi} \in L^{\prime}, \bar{\pi} \neq 0$, such that $(\bar{x}, \bar{\pi})$ is a non-trivial quasi-equilibrium of $\mathcal{E}$. Moreover, under some additional irreducibility assumption on $\mathcal{E}, \bar{x} \in$ $\mathcal{W}(\mathcal{E}) \subset \mathcal{C}^{f}(\mathcal{E})$ and, hence, $\mathcal{C}^{f}\left(\mathcal{E}^{\Gamma}\right) \subset \mathcal{C}^{f}(\mathcal{E})$.

Proof. We have already seen that the economy $\mathcal{E}^{\Gamma}$ satisfies the assumptions A.1A.5. Moreover, it follows from A.9, A.10 and Proposition 3.2 that each $Q_{i}$ is $E$ proper at $\bar{x}_{i}$ relative to $L$, with a properness vector $v_{i}$ satisfying $\bar{x}_{i}+v_{i} \in L(\omega)_{+}$.

In view of Proposition 4.2, let $\bar{\pi} \in L^{\prime}, \bar{\pi} \neq 0$, be such that $(\bar{x}, \bar{\pi})$ is a non-trivial quasi-equilibrium of $\mathcal{E}^{\Gamma}$. Then, for each $i \in I, x_{i} \in P_{i}\left(\bar{x}_{i}\right) \Longrightarrow$ $x_{i} \in \overline{\left(P_{i}\left(\bar{x}_{i}\right)+\Gamma_{i}\right) \cap L_{+}}=\overline{Q_{i}\left(\bar{x}_{i}\right)^{\tau}} \Longrightarrow \bar{\pi} \cdot x_{i} \geq \bar{\pi} \cdot \omega_{i}$. We already know that $\bar{\pi} \cdot x_{i}=\bar{\pi} \cdot \omega_{i}, \forall i \in I$ and that $(\bar{x}, \bar{\pi})$ is non-trivial. Finally, under some additional irreducibility assumption on $\mathcal{E}, \bar{x} \in \mathcal{W}(\mathcal{E})$ and it follows from the definitions that $\bar{x} \in \mathcal{C}^{f}(\mathcal{E})$.

Obviously, Proposition 4.3 and Proposition 4.4 together prove that under Assumptions A.1 - A.6, A.9, A.10 and some additional irreducibility assumption, the economy $\mathcal{E}$ has a Walrasian equilibrium.

It should also be noticed that in Assumption A.9, there is no loss of generality to assume that for each $i \in I$, the uniform properness vector $v_{i}$ satisfies $0<$ $v_{i} \leq \omega$. Moreover, in case of monotonicity of preferences $\left(\forall i \in I, \forall x_{i} \in L_{+}\right.$, $\left.P_{i}\left(x_{i}\right)+L_{+} \subset P_{i}\left(x_{i}\right)\right)$, as in Marakulin [1995], there is no loss of generality to assume that $\omega$ is the common uniform properness vector of every consumer.

\section{Concluding remarks}

Let us first compare the decentralization results proved in this paper with similar ones got in Florenzano [1990] (see Proposition 8), in case of uniform properness of preferences and under the stronger assumption that the commodity space $L$ is a locally convex-solid Riesz space.

It is worth noticing that uniform Mas-Colell properness of (strict) preferences of consumers, that is $F$-properness on $L_{+}$, implies their uniform $M$-properness, if these preferences are assumed to be asymmetric. Then, Proposition 4.4, which shows that some elements of $\mathcal{C}^{f}(\mathcal{E})$ can be decentralized as quasi-equilibria of $\mathcal{E}$ is comparable to Proposition 8 in Florenzano [1990]. Moreover in a transitive context, as in Florenzano [1990], a slightly weakened assumption of uniform 
properness of preferences is, in view of Proposition 3.1, enough to insure a full decentralization of all elements of $\mathcal{C}^{f}(\mathcal{E})$. In one case as in the other one, the conclusions of Proposition 8 in Florenzano [1990] are extended to economies defined on a commodity space $L$ verifying A.1 and A.2, at the cost of assuming that the properness vectors belong to $L(\omega)_{+}$(a restriction which disappears if $L(\omega)$ is $\tau$-dense in $L)$. In counterpart, Proposition 4.4 in this paper is got without any disposal assumption while Proposition 8 in Florenzano [1990], applied to an exchange economy, requires enough disposal to fit with a properness assumption on the disposal set considered as a production set ${ }^{4}$.

Actually, under the stronger assumption that the commodity space is locally convex-solid, free-disposal variants of Proposition 4.2 and 4.3 could easily be proved. For the variant of Proposition 4.3, it would not be necessary to assume that the uniform properness vectors belong to $L(\omega)_{+}$, so that, for this coreequivalence theorem, this restriction appears as an altenative to free-disposal, obviously related with the technique of proof.

\section{Appendix}

\section{Extension of $\bar{p}$ in the proof of Proposition 4.2}

We first begin with a lemma.

Lemma 6.1 Let $(L, \tau)$ be an ordered topological vector space, let $M$ be a vector subspace of $L$, let $Y$ be an open and convex subset of $L$ such that $Y \cap M_{+} \neq \emptyset$ and let $y \in \bar{Y} \cap M_{+}$. If $p$ is a linear functional on $M$ verifying

$$
p \cdot y \leq p \cdot z \forall z \in Y \bigcap M_{+}
$$

then there exists a $\tau$-continuous linear functional on $L$ such that $\pi_{\mid M} \leq p$ and

$$
p \cdot y=\pi \cdot y \leq \pi \cdot z \forall z \in Y \text {. }
$$

Proof. Let $Z=\left\{\left(y^{\prime}, r\right) \mid y^{\prime}=z-m, r>p \cdot m, z \in Y, m \in M_{+}\right\}$. From the assumptions of the lemma, it follows that $Z$ is an open and convex subset of $L \times R$ and that $(0, p \cdot y) \notin Z$. By the classical separation theorem, there exists $(\pi, t) \in L^{\prime} \times R$ such that

$$
t p \cdot y<\pi \cdot z-\pi \cdot m+t r, \forall z \in Y, \forall m \in M_{+}, \forall r>p \cdot m .
$$

It is easily seen that $t>0$, so that, without loss of generality, we can assume $t=1$, i.e.

$$
p \cdot y \leq \pi \cdot z-\pi \cdot m+p \cdot m, \forall z \in Y, \forall m \in M_{+} .
$$

\footnotetext{
${ }^{4}$ A production set $Y=-L_{+}$satisfies uniform properness for production as formulated by Richard [1986], while $Y=\{0\}$ does not verify this assumption.
} 
Since $y \in \bar{Y}$, we get $(p-\pi) \cdot m \geq(p-\pi) \cdot y, \forall m \in M_{+}$, which implies $(p-\pi) \cdot m \geq 0$, $\forall m \in M_{+}$, i.e. $p-\pi_{\mid M} \geq 0$. Letting $m=0$, we get $\pi \cdot y \leq p \cdot y \leq \pi \cdot z, \forall z \in Y$ and, since $y \in \bar{Y}, \pi \cdot y \leq p \cdot y \leq \pi \cdot y$, i.e. $\pi \cdot y=p \cdot y$.

We now consider the first case in the statement of Proposition 4.2 :

- $L(\omega)$ is $\tau$-dense in $L$ and each $P_{i}$ is $F$-proper at $\bar{x}_{i}$, with a properness vector $v_{i}$ satisfying $\bar{x}_{i}+v_{i} \in L_{+}$.

Recall that $(\bar{x}, \bar{p})$ is a quasi-equilibrium of $\mathcal{E}_{\mid L(\omega)}$. In view of the $\tau$-density in $L$ of $L(\omega)$, our aim here is to prove that, under the previous properness assumptions, $\bar{p}$ is actually $\tau$-continuous on $L(\omega)$.

In order to apply the previous lemma, let us define for each $i, Y_{i}=\bar{x}_{i}+\Gamma_{i}$ (where $\Gamma_{i}$ is the properness cone $\Gamma_{i}=\left\{\lambda\left(v_{i}-u\right) \mid \lambda>0, u \in V_{i}\right\}$ ) and set $y_{i}=\bar{x}_{i}, M=L(\omega), p=\bar{p}$. $Y_{i}$ is open and convex. We have already seen that $\left(\bar{x}_{i}+\Gamma_{i}\right) \cap L(\omega)_{+} \neq \emptyset$. We now verify that $\bar{p} \cdot \bar{x}_{i} \leq \bar{p} \cdot z, \forall z \in\left(\bar{x}_{i}+\Gamma_{i}\right) \cap L(\omega)_{+}$. Indeed, let $z \in\left(\bar{x}_{i}+\Gamma_{i}\right) \cap L(\omega)_{+}$. For $\lambda>0$ and small enough, $\bar{x}_{i}+\lambda\left(z-\bar{x}_{i}\right) \in$ $P_{i}\left(\bar{x}_{i}\right) \cap L(\omega)_{+}$and $\bar{p} \cdot\left(\bar{x}_{i}+\lambda\left(z-\bar{x}_{i}\right)\right) \geq \bar{p} \cdot \omega_{i}=\bar{p} \cdot \bar{x}_{i}$. Hence $\bar{p} \cdot z \geq \bar{p} \cdot \bar{x}_{i}$. Applying Lemma 5.1, we get for each $i$ a $\tau$-continuous linear functional $\pi_{i}$ on $L$ such that $\pi_{i \mid L(\omega)} \leq \bar{p}$ and

$$
\bar{p} \cdot \bar{x}_{i}=\pi_{i} \cdot \bar{x}_{i} \leq \pi_{i} \cdot z, \forall z \in \bar{x}_{i}+\Gamma_{i} .
$$

Let us define $\bar{\pi}=V_{i} \pi_{i}$. It follows from Assumption A.2 that $\bar{\pi} \in L^{\prime}$. From $\sum_{i \in I} \bar{x}_{i}=\omega$, one deduces $\bar{p} \cdot \omega \leq \bar{\pi} \cdot \omega$ and, in view of $\bar{\pi}_{\mid L(\omega)} \leq \bar{p}, \bar{p} \cdot \omega=\bar{\pi} \cdot \omega$. Now, the positive linear functional $\left(\bar{\pi}_{\mid L(\omega)}-\bar{p}\right)$ satisfies $(\bar{\pi}-\bar{p}) \cdot \omega=0$. As $\omega$ is quasi-interior in $L(\omega)_{+}, \bar{\pi}$ coincides with $\bar{p}$ on $L(\omega)$. This show that $p$ is $\tau$ continuous on $L(\omega)$. Moreover, in view of the $\tau$-density in $L$ of $L(\omega), \bar{\pi}$ is the unique $\tau$-continuous extension to $L$ of $\bar{p}$ and $(\bar{x}, \bar{\pi})$ is a quasi-equilibrium of $\mathcal{E}$.

We turn now to the second case:

- each $P_{i}$ is $E$-proper at $\bar{x}_{i}$ relative to $L(\omega)$, with a properness vector satisfying $\bar{x}_{i}+v_{i} \in L(\omega)_{+}$.

We define for each $i \in I, Y_{i}=P_{i}\left(\bar{x}_{i}\right)+\Gamma_{i}$ and set $y_{i}=\bar{x}_{i}, M=L(\omega)$, $p=\bar{p} . Y_{i}$ is open and convex. That $\left(P_{i}\left(\bar{x}_{i}\right)+\Gamma_{i}\right) \cap L(\omega)_{+} \neq \emptyset$ follows easily from $\bar{x}_{i}+\lambda v_{i} \in P_{i}\left(\bar{x}_{i}\right) \cap L(\omega)_{+}$for every positive and small enough $\lambda$ and obviously $\bar{x}_{i} \in \overline{P_{i}\left(\bar{x}_{i}\right)+\Gamma_{i}} \cap L(\omega)_{+}$. Applying Lemma 6.1 , let for each $i, \pi_{i} \in L^{\prime}$ be such that $\pi_{i \mid L(\omega)} \leq \bar{p}$ and

$$
\bar{p} \cdot \bar{x}_{i}=\pi_{i} \cdot \bar{x}_{i} \leq \pi_{i} \cdot x_{i}, \forall x_{i} \in P_{i}\left(\bar{x}_{i}\right)+\Gamma_{i} .
$$

Let us define $\bar{\pi}=\bigvee_{i} \pi_{i}$. As previously, $\bar{\pi} \in L^{\prime}, \bar{p} \cdot \omega \leq \bar{\pi} \cdot \omega$ and $\bar{\pi}$ coincides with $\bar{p}$ on $L(\omega)$. Moreover, $x_{i} \in P_{i}\left(\bar{x}_{i}\right) \Longrightarrow \bar{\pi} \cdot x_{i} \geq \pi_{i} \cdot x_{i} \geq \bar{p} \cdot \bar{x}_{i}=\bar{\pi} \cdot \bar{x}_{i}$, which shows that $(\bar{x}, \bar{\pi})$ is a quasiequilibrium of $\mathcal{E}$. 


\section{References}

[1] Aliprantis C.D. , K.C. Border and O. Burkinshaw (1997): "Economies with many commodities", Journal of Economic Theory 74, 62105.

[2] Aliprantis C.D. ,D.J. Brown and O. Burkinshaw (1987): "Edgeworth equilibria", Econometrica 55, 1109-1137.

[3] Aliprantis C.D. ,D.J. Brown and O. Burkinshaw (1990): "Existence and Optimality of Competitive Equilibria", Springer Verlag, New York.

[4] Araujo A. and P.K. Monteiro (1989): "Equilibrium without uniform conditions", Journal of Economic Theory 48, 416-427.

[5] Aubin J.P. (1979): "Mathematical Methods of Game and Economic Theory", North Holland, Amsterdam/New York/Oxford.

[6] Bewley T.F. (1972): "Existence of equilibria in economies with infinitely many commodities", Journal of Economic Theory 4, 514-540.

[7] Debreu G. AND H. Scarf (1963): A limit theorem on the core of an economy", International Economic Review 4, 235-246.

[8] DuffiE D. AND W.R. ZAME (1989): "The consumption based capital asset pricing model", Econometrica 57, 1274-1298.

[9] Florenzano M. (1982): "The Gale-Nikaido-Debreu lemma and the existence of transitive equilibrium with or without the free-disposal assumption", Journal of Mathematical Economics 9, 113-134.

[10] Florenzano M. (1990): "Edgeworth equilibria, fuzzy core and equilibria of a production economy without ordered preferences", Journal of Mathematical Analysis and Applications 153, 18-36.

[11] Mas-COLELl A. (1986): "The price equilibrium existence problem in topological vector lattices", Econometrica 54, 1039-1054.

[12] Mas-Colell A. AND S.F. Richard (1991): "A new approach to the existence of equilibria in vector lattices", Journal of Economic Theory 53, 1-11.

[13] Mas-Colell A. AND W.R. Zame (1991): "Equilibrium theory in infinite dimensional spaces", in "Handbook of Mathematical Economics" (W. Hildenbrand and H. Sonnenschein, Eds.), Vol. 4, North Holland, New York.

[14] Marakulin V.M. (1995): "An equilibrium in vector lattices: the nontransitive case and overlapping generations models", Preprint, Institute of Mathematics, Novosibirsk. 
[15] Podczeck K. (1996): "Equilibria in vector lattices without ordered preferences or uniform properness", Journal of Mathematical Economics 25, $465-485$.

[16] Richard S.F (1986): "Competitive equilibria in Riesz spaces", mimeographed G.S.I A., Carnegie Mellon University.

[17] RichaRD S.F (1989): "A new approach to production equilibria in vector lattices", Journal of Mathematical Economics 18, 41-56.

[18] Yannelis N.C. AND W.R. Zame (1986): "Equilibria in Banach lattices without ordered preferences", Journal of Mathematical Economics 15, 85110 .

[19] ZAME W.R. (1987): "Competitive equilibria in production economies with an infinite-dimensional commodity space", Econometrica 55, 1075-1108. 